\title{
Simulating an X-ray Polarimeter Gas Pixel Detector
}

\author{
Aera JUNG* \\ Department of Engineering physics, Tsinghua University, Beijing China \\ E-mail: erajung@gmail.com
}

\begin{abstract}
X-ray polarimetry is one of the last remaining largely untouched frontiers in observational astronomy and provides a novel way to probe poorly understood details of high energy emission processes for a variety of astrophysical sources. X-ray polarization is exceedingly difficult to measure, one solution is the Gas Pixel Detector (GPD). In a GPD an astronomical X-ray enters a gas cell, collides with an atom of gas, which emits a high energy electron. Critically, the direction of this electron corresponds to the polarization of the astronomical X-ray. This electron creates an ionization tract whose electrons are drifted by a small electric potential across a gas cell on to a bottom plate consisting of a double layered conductor separated by an insulator with a strong potential difference between them. This bottom plate, called a Gas Electron Multiplier (GEM), has an array of tiny holes and the ionization tract electrons fortunate enough to pass though the holes are strongly accelerated causing them to create secondary cascades in the direction of a pixelated ASIC detector array. The cumulative pattern of secondary shower electrons across the detector pixels thus reflects the direction of the initial electron ejected from the collision with the astronomical X-ray and thus ultimately the polarization of the astrophysical X-ray source.

Therefore, for effective detector operation, it is necessary to characterize the detector's response to varying internal physical properties such as pressure, temperature, and voltage configurations both to determine optimal parameters for launch and to understand changes in post-launch detector performance. Here I will discuss my work simulating the secondary cascade electron behavior using the Garfield++ software package, developed by CERN for modeling particle detectors with gas particle interactions such as drift chambers.
\end{abstract}

36th International Cosmic Ray Conference -ICRC2019-

July 24th - August 1st, 2019

Madison, WI, U.S.A.

${ }^{*}$ Speaker. 


\section{Introduction}

There are many objects in the universe such as black holes, pulsars, and supernova remnants which emit X-rays. While the nature of the X-ray emission process is often understood (e.g. inverse Compton scattering), the configuration and geometry of the emitting systems, especially their magnetic fields, often remains mysterious. X-rays, like all electromagnetic radiation, can be observed in four fundamentally different ways: imaging, spectroscopy, photometry, and polarimetry. This provides information on the spatial, spectral, timing, and polarization properties of the emission source each of which provides a unique insight into the underlying object [1].

In particular, polarization gives us information not only on the geometry of the emitting material but also of the magnetic and gravitational fields present in the emission vicinity. The angle of polarization is the orientation of the plane of vibration in the electric vector of the incoming photons, thus it is dependent on the geometry, particularly the inclination, of the emitting process. The degree of polarization is a quantitive measure of the fraction of light which is polarized, thus it is dependent on the degree and type of symmetry in the emitting system. Therefore, without polarimetry our observations are incomplete as we cannot fully harness the information in the light we are receiving [2].

Unfortunately, while polarimetry has proved very important in the radio, IR, and optical bands (and proved critical to our understanding many physical systems such as jets in blazers), polarization observations in the X-ray band are largely nonexistent. In the 1970s, the first observations of X-ray polarization were conducted on the emission of the Crab Nebula using graphite crystal Bragg polarimeters. Unfortunately, there was no significant technical improvement for decades, limiting X-ray polarimetry observations to only a couple of the very brightest astronomical sources. Recently, this situation has dramatically changed with the advent of polarization detectors based on the photoelectric effect.

With an atom absorbs a photon with sufficient energy to eject an electron, the electric field vector of the photon drives the direction of the electron emission. Therefore the angle of the emitted electron corresponds statistically with the polarization of the incoming X-ray photon. Two different technologies are now available to perform polarimetry using the photoelectric effect $[1,2,3,4]$. One is the Gas Pixel Detector (GPD) which was developed in Italy and anther is a Time Projection Changer (TPC) which was developed in the USA.

In both cases, the X-ray photon is absorbed in a gas cell and the ionization track produced by the photoelectron is drifted and multiplied by a Gas Electron Multiplier (GEM) and eventually, read out by a detector located on an anodic plate. The difference between the GPD and TPC, is that in the GPD the incoming photon direction is perpendicular to the detector. Thus the detector is also parallel the plane of maximum probability of photoelectron emission. In the TPC the incoming photon direction is parallel to the detector. The ionization charges are drifted orthogonally with respect to the optical axis and the detector quantum efficiency can be increased simply by making a deeper gas cell. In this paper, I discuss GPD detectors exclusively.

\section{Gas Electron Multiplier (GEM)}

The single Gas Electron Multiplier (GEM) is a planar dielectric clad in metal with holes coated with a thin copper layer on each side (Fig. 1 left). It is an amplification structure independent of the readout. The GEM uses a pair of highly perforated plates on each side of an similarly perforated 
insulator to apply a strong electric field while still allowing electrons to freely enter and exit the system (Fig. 1 middle). The electrons generated by the incident X-rays are multiplied in an electron avalanche as they pass through the holes located in the GEM below. Then a readout application specific integrated circuit (ASIC) collects these signals. Multiple layers of GEMs can be stacked to provide further amplification [5, 6]. However in this paper only a single layer GEM is considered. The dielectric used is a liquid crystal polymer (LCP) with a thickness of the order $100 \mu \mathrm{m}$ with a copper layer $5 \mu \mathrm{m}$ thick deposited on both surfaces. In Fig. 1 right, we can see a person holding a GEM through the GEM itself.
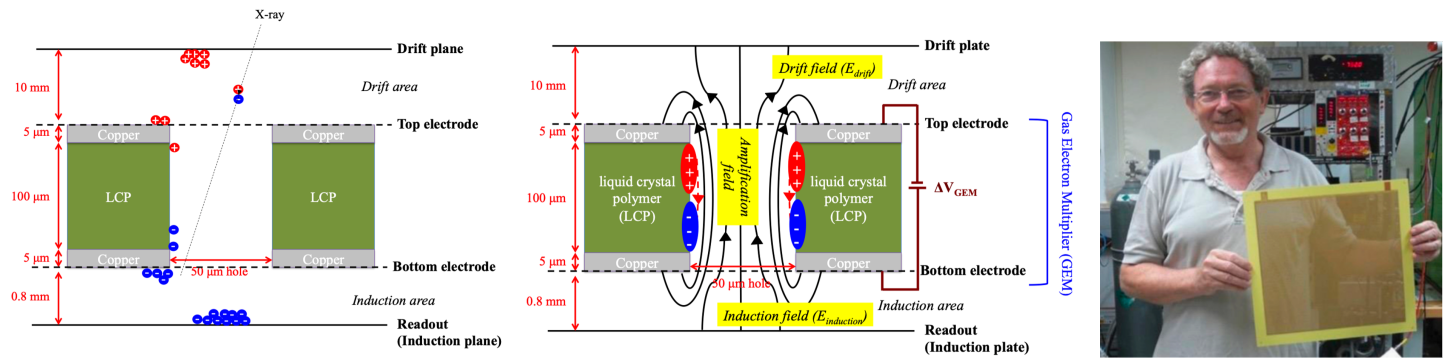

Figure 1: Left: Sketch of the charging-up process during an avalanche. Middle: Sketch of the cross section of a GEM foil with electric field lines. Right: GEM foil showing its transparency (Originally from [4]).

Figure 2 shows an avalanche initiated by a single electron in dimethyl ether (DME) gas for various sizes of copper etching. The electron transport is modeled using a Monte Carlo simulation in the Garfield++ simulation tool. While we need many events to build up a polarization measurement, for simplicity, this figure shows only a single event. A comparison of electron losses vs. different sizes of copper etching is discussed in section 3.2.2.

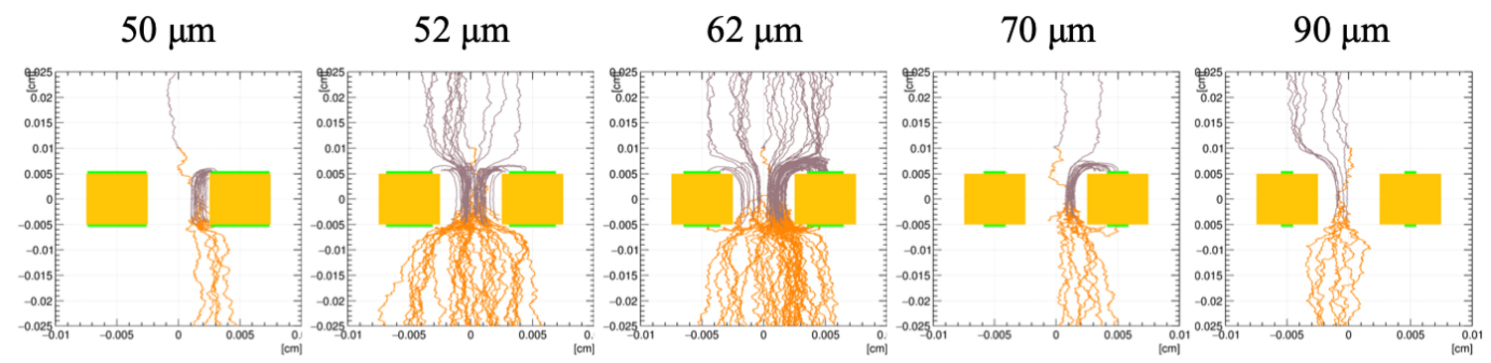

Figure 2: An avalanche initiated by a single electron in dimethyl ether (DME) gas is shown for several different sizes of copper etching. The drift field was $\sim 2.1 \mathrm{kV} / \mathrm{cm}$ and the induction field was $\sim 4.7 \mathrm{kV} / \mathrm{cm}$ at $\mathrm{p}=1 \mathrm{~atm}$ and $\mathrm{T}=20^{\circ} \mathrm{C}$. (The drift field and induction field are above and below the GEM as shown in Fig $1)$.

The following are the GEM physical parameters used in the simulations along with their definitions [7, 8]:

- Top electrode: The copper metal layer of a GEM to which the lower potential is applied. This is the GEM electrode closer to the drift plate.

- Bottom electrode: The copper metal layer of a GEM to which the higher potential is applied. This is the GEM electrode closer to the induction plate.

- Thickness of top electrode: The thickness of the top electrode (i.e. conductive) surface of the GEM.

- Thickness of bottom electrode: The thickness of the bottom electrode. Usually this is the same as the top electrode. 
- GEM hole: Diameter of the holes in the dielectric material.

- Copper coating hole: Diameter of the hole in the metal constituting the top and bottom GEM electrodes.

- GEM hole Pitch: Pitch is the distance between the centers of GEM holes.

- Drift plate distance: The distance between the drift plate and the top of the GEM. The drift plate is the top of the GEM detector and is a metal sheet with the lowest electric potential in the system, thus acting as a cathode to provide a field which drifts the electrons down through the gas cell towards the GEM. The region enclosed between the drift plane and the upper electrode is the drift space (region / chamber) where the primary charge is generated in the ionization track caused by the incident ionizing X-ray radiation.

- Induction plate distance: The distance between the bottom of the GEM and the induction plate holding the readout ASIC. The induction plate is the bottom of the GEM detector, consisting of a copper metal plate with the highest potential in the system, thus acting as an anode to attract electrons generated in the GEM. Usually it is kept at the ground potential of the electronics because the readout ASIC is imbedded within the induction plate. The region between the lower GEM electrode and the induction plate is called the induction gap.

- Gain: The gain is defined as the average number of electrons reaching the anode per primary electron.

- Primary charge: Charge generated in the drift chamber by ionization due to the initial electron emitted by the photoelectric effect from the incident radiation.

- Secondary charge: Charge due to avalanche multiplication under the influence of the applied GEM electric field.

\section{Simulation software and results}

The simulation is performed by varying the characteristics of the detector. Here I will focus on simulating the gain by varying the electron flow into the top of the GEM. Therefore we will focus primarily on changing the GEM copper coating hole diameter and the drift plate distance.

\subsection{Software}

First, the physical geometry of the GEM detector is created using a software package called Gmsh [9] using the values described in section 2. Next, the physical model created by Gmsh is inputted into another software package called Elmer [10]. Also inputed into Elmer are the electric potentials for the conductive components of the GEM detector, primarily the drift plate and upper GEM electrode voltages. Elmer then creates an electric field model of the system which is then fed into the Garfield++ [8] simulation tool. Garfield++ is then used to simulate the electron transportation in the GEM detector.

\subsubsection{Garfield++}

Garfield and Garfield++ are a computer simulation packages developed by CERN for modeling particle detectors with gas particle interactions such as drift chambers. Garfield is the original version written in Fortran and Garfield++ is a newer version using C++. Of course, Garfield++ shares functionality with Garfield and is built on the ROOT (a data analysis program developed by CERN) [11] platform with allows the user to easily view the drift lines of electrons and ions, and also to do basic filtration of data by adding user-made functions. The main differences are the more up-to-date treatment of electron transport in gases and the user interface, which is derived 
Table 1: Input parameters for a single GEM simulation

\begin{tabular}{cccc}
\hline Description & Default Value & Material & Value Varations \\
\hline \hline diameter of GEM hole & $50 \mu \mathrm{m}$ & \\
diameter of copper etching & $50 \mu \mathrm{m}$ & & $50,52,62$, \\
around GEM hole & & & $70,82,90 \mu \mathrm{m}$ \\
thickness of GEM dielectric & $100 \mu \mathrm{m}$ & $\begin{array}{c}\text { liquid crystal } \\
\text { polymer (LCP) } \\
\text { copper }\end{array}$ \\
thickness of top/bottom GEM electrode & $5 \mu \mathrm{m}$ & dimethyl ether & $1,2 \mathrm{~cm}$ \\
GEM hole pitch & $100 \mu \mathrm{m}$ & \\
drift plate distance & $1 \mathrm{~cm}$ & \\
(DME) gas & \\
induction plate distance & $0.08 \mathrm{~cm}$ & & \\
voltage between top and bottom plate & $691 \mathrm{~V}$ & \\
drift field & $2132 \mathrm{~V} / \mathrm{cm}$ & \\
induction field & $4712.5 \mathrm{~V} / \mathrm{cm}$ &
\end{tabular}

from ROOT. Unfortunately, Garfield and Garfield++ do not support the same range of input file types thus limiting us to using the Gmsh to Elmer to Garfield++ progression.

\subsubsection{Gmsh and Elmer}

Because Garfield++ can not compute more complex electric fields, the free software Gmsh and Elmer are used in the simulation. Gmsh creates a bottom-up geometry for the GPD detector and provides mesh-like post-processing. Elmer is an open source multiphysical simulation tool that can be used to solve the electric field equations and create a model of the electric potential throughout the GPD. The required meshes are created in Gmsh and solved using a finite element method in Elmer. Because Linux programs can be interconnected, you can use a single script to create the field file you need to input into Garfield++, since you set the voltage on the geometry and electrodes at the same time.

\subsection{Results}

In this section, the results of my studies are briefly reported using the geometry parameters of the GEM which are shown in table 1. The study of gain for variable voltage, pressures, and temperature changes can be seen in subsection 3.2.1. Also, the study of gain for different copper size is shown in subsection 3.2.2. Finally, an electric field study is introduced in subsection 3.2.3.

\subsubsection{Gain}

Gain is defined as the average number of electrons reaching the anode per primary electron. Gain varies with almost every parameter involved in the operation of a GPD. In this paper, these include pressure, temperature and electric field. Here we have performed a number of simulations in order to identify how to optimize these parameters for detector operation. For these gain simulations, the drift plate and induction plate distances remain fixed at $1 \mathrm{~cm}$ and $0.8 \mathrm{~mm}$ respectively.

Here we simulate the detector gain while varying the temperature from 0 to $30{ }^{\circ} \mathrm{C}$ at increments of $5^{\circ} \mathrm{C}$, the pressure from 0.6 to $1 \mathrm{~atm}$ at increments $0.05 \mathrm{~atm}$ and across a range of voltages. The left side of Fig. 3 shows the results of various temperatures and pressures. The middle figure is a replot of the left with a different scaling on the ordinate so as to better show the high pressure behavior. As you can see, the gain increases with temperature but, decreases with increasing pressure. 
Table 2: Detailed voltage information for Fig. 3 right

\begin{tabular}{cccc}
\hline Color & $\Delta V_{G E M}[V]$ & Drift field $\left(E_{\text {drift }}\right)[\mathrm{V} / \mathrm{cm}]$ & Induction field $\left(E_{\text {induction }}\right)[\mathrm{V} / \mathrm{cm}]$ \\
\hline \hline black & 604.24 & 1865.64 & 4126.5 \\
red & 625.82 & 1932.27 & 4273.875 \\
green & 647.4 & 1998.9 & 4421.25 \\
blue & 668.98 & 2065.53 & 4568.625 \\
yellow & 690.56 & 2132.16 & 4716 \\
magenta & 712.14 & 2198.79 & 4863.375 \\
cyan blue & 733.72 & 2265.42 & 5010.75 \\
\hline
\end{tabular}

The GPD contains four sets of conductive plates with different voltages: a bottom induction plate with a potential defined as the ground, the bottom and top electrodes on the GEM, and the drift plate at the top of the detector. Here we simulate different detector voltages by uniformly increasing the electric potential throughout the detector. The gain as a function of pressure for different voltages are shown in the Fig. 3 right using different colors. Table 2 shows the detailed voltages at different locations in the detector for each of these colors. As the voltage difference increases the gain increases, however, the difference in gain due to the voltage difference is not as large at high pressure. However, the highest gain is not always optimal. The secondary electrons are produced the GEM and arrive at the ASIC readout plate. Through ASIC data, we can analyses different angular directions and detect photons with respect to these directions. If gain is too high, the number of arriving electrons exceeds the ability of the detector software to preform the reconstruction, thus degrading the accuracy of measuring the angular direction of the primary electron track and ultimately the polarization of the incoming X-rays.
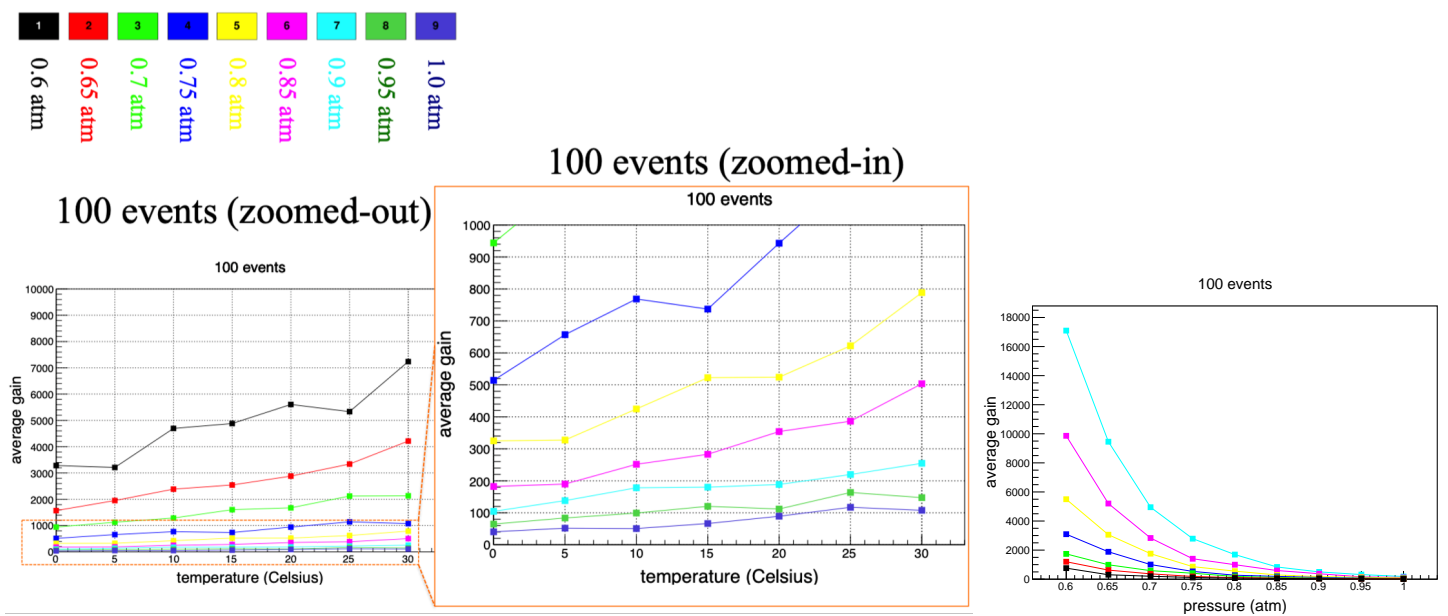

Figure 3: Left: average gain as a function of temperature and pressure, Middle: zoomed-in left panel to better show the high pressure behavior, Right: average gain as a function of pressure and voltage

\subsubsection{Losses of secondary charges}

As the GEM is an electrode plate with holes in it, not all primary electrons enter a hole and not all the secondary electrons created in the GEM exit it successfully. Usually, the main loss points for primary electrons are colliding with the top electrode of the GEM and secondary electron losses are colliding with the bottom GEM electrode (there are also some losses due to collisions with the side of the GEM insulator). Secondary electron losses can be reduced by increasing the induction field 
(the potential between the bottom of the GEM and the induction plate). In addition, loss occurs inside the GEM holes due to electrons diffusing from the gas inside the GEM holes. Due to the high electron affinity of the dielectric, electrons can not diffuse back into the gaseous medium once they become attached to the dielectric surface. In addition to the GEM hole, these losses can also happen in the drift and induction gaps.

The extended study of the gain simulates where secondary charges stop and how many of the generated secondary charges arrive at the readout ASIC. For the purposes of this simulation, the number of electrons reaching $0.3 \mathrm{~mm}$ above the readout ASIC are counted as having arrived and used to compute the gain.

The diameter of the GEM hole was kept at $50 \mu \mathrm{m}$ as the thickness of the dielectric and top/bottom electrode, hole pitch, distance between drift and induction plates, and the drift and induction electric fields were also fixed. The diameter of the copper GEM electrode holes was symmetrically changed to 50, 52, 62, 70, 82, and $90 \mu \mathrm{m}$. This is shown in Fig. 2 with the of copper etching in green and dielectric in yellow.

In Fig. 4 left, the green box shows the percentage of electrons reaching the bottom of the GEM plate and the yellow box shows the percentage of electron reaching the ASIC readout. The larger the diameter of the copper metal hole, the more electrons arrive at the readout ASIC.
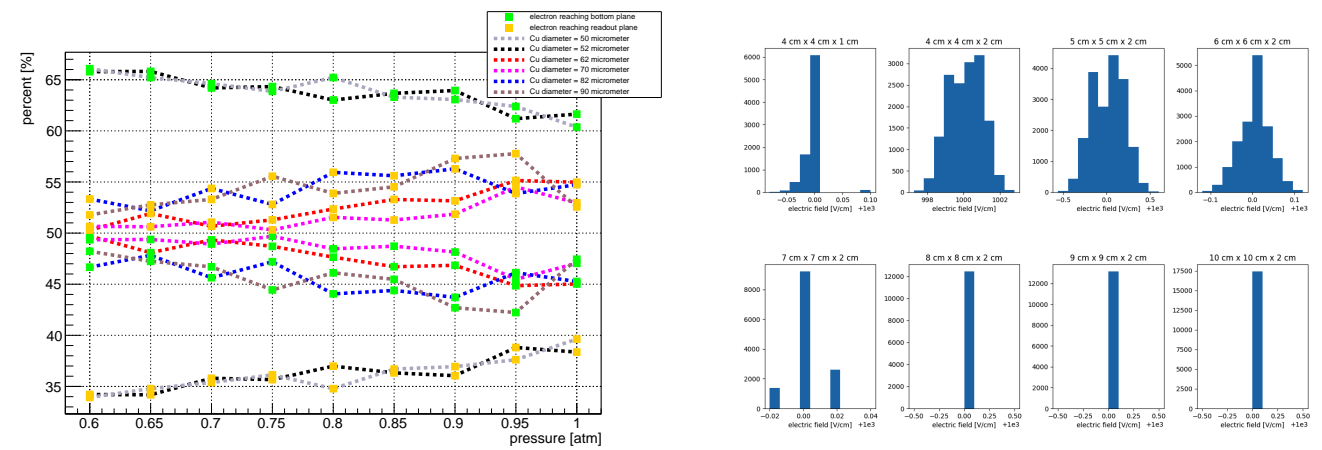

Figure 4: Left: Percentage of secondary electrons that reach the GEM plate (green box) and readout ASIC (yellow box) as a function of pressure, Right: Histogram of drift chamber mesh volume electric field for different drift chamber geometries. This is obtained by calculating the electric field (regardless of orientation) of each simulation mesh element and counting the number of mesh elements within each electric field range bin.

\subsubsection{Electric field}

There are 3 different electric fields in the GPD detector (see Fig. 1 middle). First is the drift field between the drift plate and the top of the GEM. Second is the amplification filed within the perforations of the GEM. Third is the induction field between the bottom of the GEM and the readout ASIC.

The drift field brings the primary electrons generated in the drift chamber down to the upper GEM electrode. The field inside the GEM hole serves to draw electrons into the hole. Therefore, a strong drift field is desirable for attracting electrons to the GEM, but a very high field can drop the field line onto the metal rather than inside the hole which reduces the gain. The induction field is set to draw secondary electrons generated in the GEM down toward the readout ASIC. 
As shown in Table 2, the induction field is stronger than the drift field. The differences in the drift field have more effect on the gain than the differences in the induction field. The size of the ASIC's effective readout area is $1.5 \mathrm{~cm} \times 1.5 \mathrm{~cm}$, and if a uniform electric field is formed inside this readout area the gain can be increased. Here we simulate the drift electric field using various shapes and various sizes for the drift chamber. The thickness of the GEM and induction chamber remain fixed.

Using the same size of parallel plate in the top and bottom of the drift chamber produced a better electric field than different sizes of parallel plates. Also, when the drift distance (the height of the drift chamber) is increased, the drift electric field was formed less uniformly. As the drift distance increases, the gain increases. We simulate various sizes for the uniform drift electric field in the effective readout area. Figure 4 right shows the electric field in the effective readout area for various drift chamber sizes. The upper left plot of Fig. 4 right shows the baseline drift chamber geometry used exclusively for the other simulations. In other words, the base model is a $4 \times 4 \times 1$ $\mathrm{cm}^{3}$ cubic. Upon increasing the drift distance to $2 \mathrm{~cm}$, we can see that the drift field is not uniform. We then change the size of the parallel plate to find a drift electric field with a uniformity similar to the baseline drift field. If the parallel plate is more than $7 \times 7 \mathrm{~cm}^{2}$, it represents a drift electric field consistency similar to the baseline model but with increased gain.

\section{References}

[1] Enrico Costa, Paolo Soffitta, Ronaldo Bellazzini, Alessandro Brez, Nicholas Lumb, and Gloria Spandre. An efficient photoelectric x-ray polarimeter for the study of black holes and neutron stars. Nature, 411:662-665, 2001.

[2] S. Fabiani. Instrumentation and Future Missions in the Upcoming Era of X-ray Polarimetry. Galaxies, 6:54, May 2018.

[3] Martin C. Weisskopf. X-Ray Polarimetry: Historical Remarks and Other Considerations. 2009.

[4] Fabio Sauli. The gas electron multiplier (gem): Operating principles and applications. $\mathrm{Nu}$ clear Instruments and Methods in Physics Research Section A: Accelerators, Spectrometers, Detectors and Associated Equipment, 805:2 - 24, 2016. Special Issue in memory of Glenn F. Knoll.

[5] P. M. M. Correia, M. Pitt, C. D. R. Azevedo, A. Breskin, S. Bressler, C. A. B. Oliveira, A. L. M. Silva, R. Veenhof, and J. F. C. A. Veloso. Simulation of gain stability of THGEM gas-avalanche particle detectors. JINST, 13(01):P01015, 2018.

[6] Andrej Sipaj. Simulation, Design and Construction of a Gas Electron Multiplier for Particle Tracking. PhD thesis, 2012.

[7] Mythra Varun Nemallapudi. Gain of a single gas electron multiplier, 2012.

[8] CERN. Garfield++, https://garfieldpp.web.cern.ch/garfieldpp/.

[9] Christophe Geuzaine and Jean-François Remacle. A three-dimensional finite element mesh generator with built-in pre- and post-processing facilities, http://gmsh.info/.

[10] CSC IT Center for Science. Elmer, https://www.csc.fi/web/elmer.

[11] CERN. Root data analysis framework, https://root.cern.ch. 